\title{
A Novel Therapeutic Approach to Corneal Alkaline Burn Model by Targeting Fidgetin-Like 2, a Microtubule Regulator
}

\author{
Jessie Wang ${ }^{1,2}$, Abhinav Dey ${ }^{2}$, Adam H. Kramer ${ }^{2}$, Yuan Miao ${ }^{1}$, Juan Liu ${ }^{1}$, Lisa Baker², \\ Joel M. Friedman ${ }^{3}$, Parimala Nacharaju ${ }^{3}$, Roy S. Chuck ${ }^{1}$, Cheng Zhang ${ }^{1, *}$, and \\ David J. Sharp ${ }^{1-4, *}$
}

\author{
${ }^{1}$ Department of Ophthalmology \& Visual Sciences, Montefiore Medical Center, Albert Einstein College of Medicine, Bronx, NY, USA \\ ${ }^{2}$ MicroCures, Inc., Bronx, NY, USA \\ ${ }^{3}$ Department of Physiology \& Biophysics, Albert Einstein College of Medicine, Bronx, NY, USA \\ ${ }^{4}$ Dominick P. Purpura Department of Neuroscience, Albert Einstein College of Medicine, Bronx, NY, USA
}

Correspondence: David J. Sharp, Albert Einstein College of Medicine, 1300 Morris Park Rd., Bronx, NY 10461, USA. e-mail: david.sharp@einsteinmed.org

Received: June 19, 2020

Accepted: December 11, 2020

Published: January 8, 2021

Keywords: Fidgetin-like 2; siRNA; corneal re-epithelialization

Citation: Wang J, Dey A, Kramer AH, Miao Y, Liu J, Baker L, Friedman JM, Nacharaju P, Chuck RS, Zhang C, Sharp DJ. A novel therapeutic approach to corneal alkaline burn model by targeting Fidgetin-like 2, a microtubule regulator. Trans Vis Sci Tech. 2021;10(1):17, https://doi.org/10.1167/tvst.10.1.17
Purpose: The purpose of this study was to determine the efficacy of nanoparticleencapsulated Fidgetin-like 2 (FL2) siRNA (FL2-NPsi), a novel therapeutic agent targeting the $F L 2$ gene, for the treatment of corneal alkaline chemical injury.

Methods: Eighty 12-week-old, male Sprague-Dawley rats were divided evenly into 8 treatment groups: prednisolone, empty nanoparticles, control-NPsi $(1 \mu \mathrm{M}, 10 \mu \mathrm{M}$, and $20 \mu \mathrm{M})$ and FL2-NPsi $(1 \mu \mathrm{M}, 10 \mu \mathrm{M}$, and $20 \mu \mathrm{M})$. An alkaline burn was induced onto the cornea of each rat, which was then treated for 14 days according to group assignment. Clinical, histopathologic, and immunohistochemical analyses were conducted to assess for wound healing. FL2-NPsi-mediated knockdown of FL2 was confirmed by in vitro quantitative polymerase chain reaction (qPCR). Toxicity assays were performed to assess for apoptosis (terminal deoxynucleotidyl transferase-mediated deoxyuridine triphosphate nick-end labeling [TUNEL] assay) and nerve damage (whole mount immunochemical staining). Statistical analyses were performed using Student's $t$-test and ANOVA.

Results: Compared with controls, FL2-NPsi-treated groups demonstrated enhanced corneal wound healing, with the 10 and $20 \mu \mathrm{M}$ FL2-NPsi-treated groups demonstrating maximum rates of corneal re-epithelialization as assessed by ImageJ software, enhanced corneal transparency, and improved stromal organization on histology. Immunohistochemical analysis of vascular endothelial cells, macrophages, and neutrophils did not show significant differences between treatment groups. FL2-NPsi was not found to be toxic to nerves or induce apoptosis ( $p=0.917)$.

Conclusions: Dose-response studies found both 10 and $20 \mu \mathrm{M}$ FL2-NPsi to be efficacious in this rat model. FL2-NPsi may offer a novel treatment for corneal alkaline chemical injuries.

Translational Relevance: Basic cell biology findings about the microtubule cytoskeleton were used to design a therapeutic to enhance corneal cell migration, highlighting the promise of targeting microtubules to regulate corneal wound healing.

\section{Introduction}

There are an estimated 1.8 million cases of ocular trauma in the United States every year, with burn wounds accounting for 7 to $18 \%$ of eye injuries presenting to emergency rooms, $84 \%$ of which are due to

chemical injuries. ${ }^{1}$ Alkaline chemical injuries oftentimes lead to poor visual outcomes due to delayed corneal re-epithelialization, persistent inflammation, corneal ulceration and scarring. ${ }^{2}$ Typically, they are treated with extensive flushing of the eye and topical application of corticosteroids, which target the early stages of inflammation, but have no effect on epithelial 
migration and interfere with corneal stroma repair, making the tradeoff often undesirable. ${ }^{3}$

The corneal epithelium is the outermost layer of cells of the eye and plays an essential role in maintaining the smoothness of the optical surface and the health of the corneal stroma. ${ }^{4}$ The corneal epithelium is largely maintained through the production of cells by stem cells in the limbal corneal region. During homeostasis, centripetal migration of these newly formed corneal epithelial cells moves cells through multiple corneal zones into the central cornea, turning over the epithelium every 7 to 10 days. $^{5}$ In instances of small injuries to the cornea, adjacent epithelial cells simply spread to fill the defects. ${ }^{6}$ Models of corneal scrapes and abrasions produce epithelial defects that are completely healed within 24 to 72 hours, a time course often too short for detailed comparative analysis of wound healing. However, larger corneal wounds (e.g. burns and severe trauma) cannot be covered by adjacent cells and instead require increased production and extended migration of large numbers of corneal epithelial cells from the limbus into the wound zone. Accordingly, these wounds take longer to heal, offering a valuable model for ocular wound healing studies. ${ }^{7-9}$

Current therapeutics for corneal inflammatory and epithelial wound healing have focused on amniotic membranes, topical steroids, and extracellular signaling factors, with limited success. ${ }^{10}$ Small interfering RNAs (siRNA) are non-coding RNA molecules between 20 and 25 nucleotides in length, which interfere with the expression of target genes with complementary nucleotide sequences by degrading mRNA after transcription, preventing translation. This occurs within the RNA interference (RNAi) pathway within the cell. Whereas siRNA-based therapies have shown some promise for treating a wide range of maladies, including wound closure, ${ }^{11-13}$ the effective delivery of therapeutic siRNA has proven difficult in many circumstances. The siRNA is a relatively large and negatively charged molecule, making it impermeable to cellular membranes. However, the development and refinement of siRNA delivery systems with nanoparticles is helping to circumvent this challenge. ${ }^{14,15}$ Although previous siRNA studies have shown limited success, ${ }^{16,17}$ the combination of new delivery platforms and the appropriate siRNA gene target could result in new ocular therapies.

Multiple investigations in recent years have looked into harnessing the intracellular cytoskeleton, particularly microtubules, for corneal wound healing. Hollow polymeric filaments composed of tubulin subunits, microtubules provide structural support for the cell and are an important substrate for many of the molecular motor proteins responsible for intracellular transport. Regulation of the dynamic properties of microtubules are critically important for the capacity of cells to close wounds, especially near the cell periphery. Experimental alteration of microtubule organization, dynamics, and/or post-translational modification status has been shown to have significant effects on the migration of multiple corneal cell types both in vitro and in vivo. ${ }^{18-22}$ Furthermore, patients with arthritis treated with a drug that broadly depolymerizes microtubules display significantly reduced corneal wound healing. These data highlight both the promise of targeting microtubules to regulate wound healing and the need for a better understanding of how microtubule dynamics affect cell migration. ${ }^{23,24}$

Our previous work identified Fidgetin-like 2 (FL2) as a microtubule regulator important for cell migration. Specifically, FL2 localizes to the cell edge where it selectively severs dynamic microtubules to inhibit directional cell migration. As a result, silencing FL2 with siRNA (FL2-siRNA) promotes cell motility in vitro and wound healing in animal models, such as dermal excision and burn wounds in mice. ${ }^{12,13}$

MT severing enzymes, which are members of the ATPases Associated with diverse cellular Activities (AAA+) superfamily, cause breakages in MTs by forming hexameric rings around the $\mathrm{C}$-terminal tails of tubulin and using energy from ATP hydrolysis to pull on the tails, thereby causing tubulin dimers to dissociate from the MT lattice. ${ }^{25,26}$ Through their severing activity, they regulate MT length, number, and branching, and fine-tune the dynamics of the MT cytoskeleton. ${ }^{27}$ MT severing enzymes include katanin, spastin, and the fidgetin family (fidgetin, Fidgetin-like 1 [FL1], and Fidgetin-like 2 [FL2]). FL2 is highly similar to canonical fidgetin within its catalytic AAA domain but diverges elsewhere within its polypeptide sequence and is present only in vertebrates. Our previous work has demonstrated that silencing FL2 with siRNA promotes cell motility in vitro and wound closure in animal models. ${ }^{12,13}$ Here, using a dose-response study, we report that FL2 siRNA delivered via nanoparticle technology (FL2-NPsi) can promote the repair of alkali burned corneas and reduce corneal tissue edema and scarring within 2 weeks of injury.

\section{Methods}

The study protocol was approved by the Albert Einstein College of Medicine Institutional Animal Care and Use Committee (IACUC), and was conducted in adherence to the ARVO Statement for the Use of Animals in Ophthalmic and Vision Research.

Eighty 12-week-old, male Sprague-Dawley rats were divided evenly into 8 groups: positive control 
prednisolone (prednisolone acetate, Pred Forte; Allergan, Irvine, CA), empty nanoparticles, controlNPsi $(1 \mu \mathrm{M}, 10 \mu \mathrm{M}$, and $20 \mu \mathrm{M})$ and FL2-NPsi (1 $\mu \mathrm{M}, 10 \mu \mathrm{M}$, and $20 \mu \mathrm{M}$ ), with 10 rats per group. After the animals were anesthetized with isoflurane gas and an injection of ketamine and xylazine, topical $0.5 \%$ proparacaine was applied to the right cornea before surgery. The corneal epithelium was removed with a foam tip applicator, and a chemical injury was induced on the right cornea of each rat using $4 \mathrm{~mm}$ discs of $1 \mathrm{M} \mathrm{NaOH}$-soaked filter paper, applied directly for 10 seconds. Next, the eye was washed with 3 consecutive $10 \mathrm{~mL}$ washes of sterile phosphate-buffered saline (PBS). The left eye was left uninjured and untreated to serve as a negative control. The injured eye was then treated for the subsequent 14 days according to their group assignment described above, along with an antibiotic eye drop ( $0.3 \%$ ofloxacin; Allergan), with drops administered at least 10 minutes apart from each other to prevent washout. Nanoparticles were given every other day, as siRNA has an intracellular half-life of 24 to 72 hours, ${ }^{28}$ whereas prednisone treatments were given twice daily and antibiotic eye drops administered daily. Prednisolone acetate is known to have a half-life of 30 minutes in aqueous humor and is usually dosed at 2 to 4 times, ${ }^{29}$ whereas ofloxacin has a half-life of several hours, with significant concentrations still present 6 to 24 hours after administration. ${ }^{30}$

\section{Clinical Analysis}

Clinical assessment of wound healing was conducted in a blinded fashion every other day throughout the treatment period by recording digital images of corneas with a Leica EZ4 Stereo Dissecting Microscope (10447197; , Heerbrugg, Switzerland) on days $0,2,4,6,8,10,12$, and 14 . After documenting the appearance of the corneas under normal lighting, the surface was stained with a drop of fluorescein solution (Fluress; Akorn Incorporated, Decatur, IL), enabling visualization of the corneal epithelial defect. The areas of epithelial defect were quantified using ImageJ software and subsequently expressed as a percentage of the total corneal area. The results were recorded and assessed using the Student's $t$-test and ANOVA ( $N=6$ per group). Additionally, the degree of corneal opacity was scored, noting presence and degrees of hemorrhage, edema, and neovascularization (Supplement Fig. S1).

\section{Histopathologic Analysis}

At the end of the treatment period, animals were euthanized and eyes enucleated for histopatho- logic evaluation ( $N=6$ half-corneas per group). Corneal tissue was embedded in paraffin, stained with hematoxylin and eosin (H\&E), and cut to a thickness of 5 to $7 \mu \mathrm{m}$. Analyses of the tissue specimens were conducted in a blinded fashion. To carry out the immunohistochemical analyses of the corneal tissues, 4 animals per group were sacrificed at 4 different time points: days 1, 3, 7, and 14. This timeline was selected because inflammatory cells, such as neutrophils and macrophages, are most prominent between days 2 and 4 . The following antibodies were selected and tested on half of each cornea: anti-CD31 mouse monoclonal IgG1 targeting vascular endothelial cells (BioRad, Kidlington, Oxford, England), antineutrophil rabbit polyclonal IgG targeting neutrophils (LifeSpan BioSciences, Seattle, WA), and OX42 mouse monoclonal IgG2 targeting macrophages (Santa Cruz Biotechnology, Dallas, TX). All antibodies were unconjugated and stained with a secondary, dyeconjugated antibody. Specimens were embedded in paraffin and cut to a thickness of 8 to $10 \mu \mathrm{m}$. Analysis of these specimen likewise occurred in a blinded manner.

\section{Toxicity Analysis}

Evaluation for toxicity was performed to assess for apoptosis and nerve damage. To assess for apoptosis, 9 male Sprague-Dawley rats, 1 half-cornea for each of the 8 treatment groups (prednisone, empty $\mathrm{NP}$, control-NPsi at $1 \mu \mathrm{M}, 10 \mu \mathrm{M}$, or $20 \mu \mathrm{M}$, FL2NPsi at $1 \mu \mathrm{M}, 10 \mu \mathrm{M}$, or $20 \mu \mathrm{M}$ ) and an additional uninjured/untreated contralateral eye in the same rats to serve as negative control were used. Treatment groups were wounded and treated as described previously. At the end of treatment, animals were euthanized and the eyes were collected for analysis. Corneas were trimmed around the sclerolimbal region and fixed in $4 \%$ paraformaldehyde overnight. The transferase dUTP Nick End Labeling (TUNEL) assay was performed on cryosections of rat eyes using the TUNEL apoptosis detection kit (DeadEnd Fluorometric TUNEL System; Promega, Madison, WI) according to the manufacturer's instructions. Corneal toxicity from each treatment was evaluated by determining TUNEL-positive cell density (number per square millimeter) calculated as follows: number of TUNEL-positive cells/total number of cells. Three random fields of view were taken per corneal tissue sample.

To assess for nerve damage, one halfcornea per treatment group with an additional uninjured/untreated contralateral eye from the same rats to serve as negative control (9 half-corneas in total) 


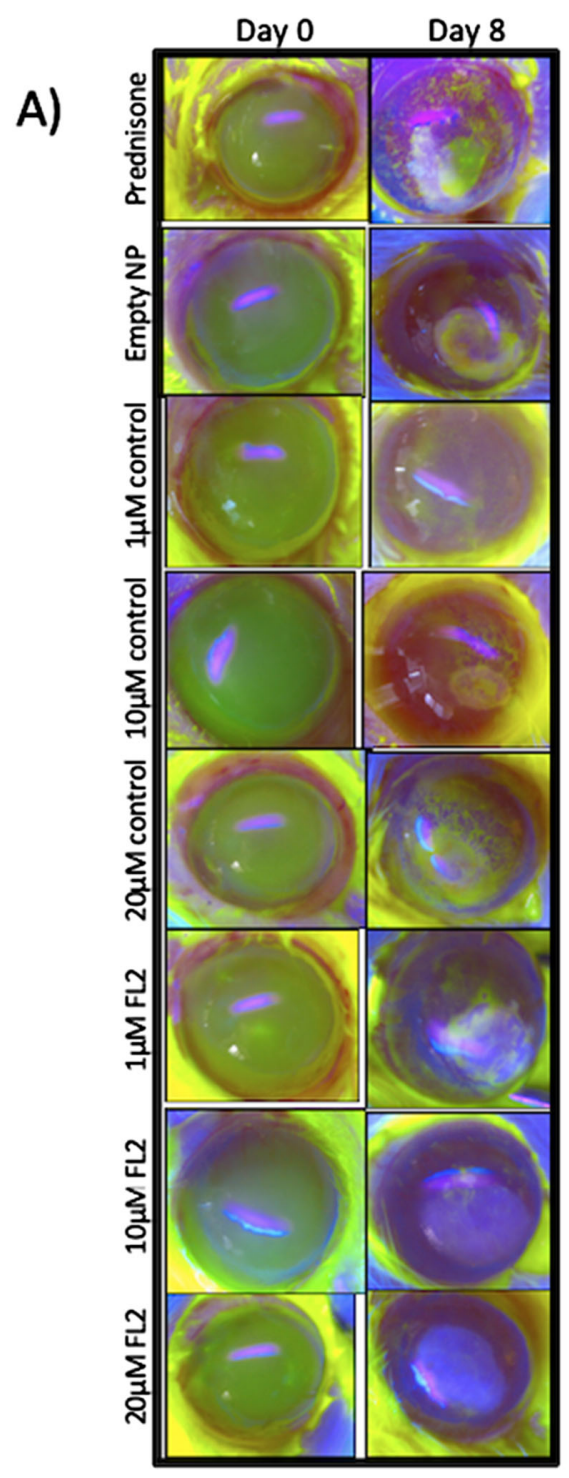

B)

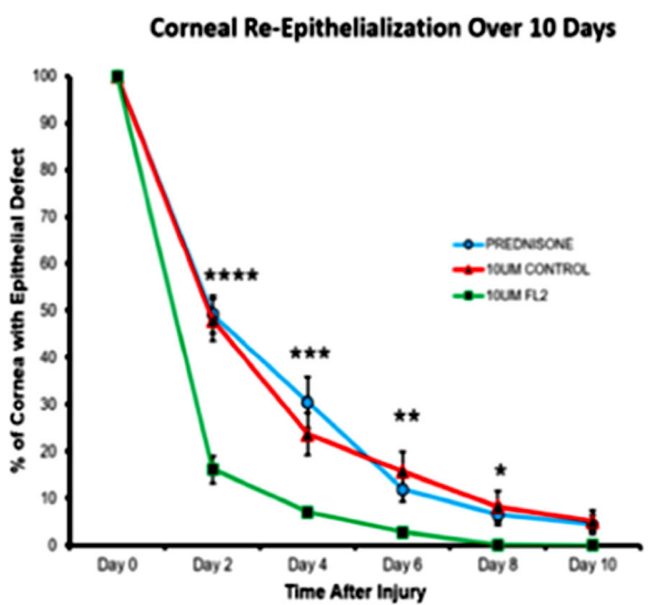

C)

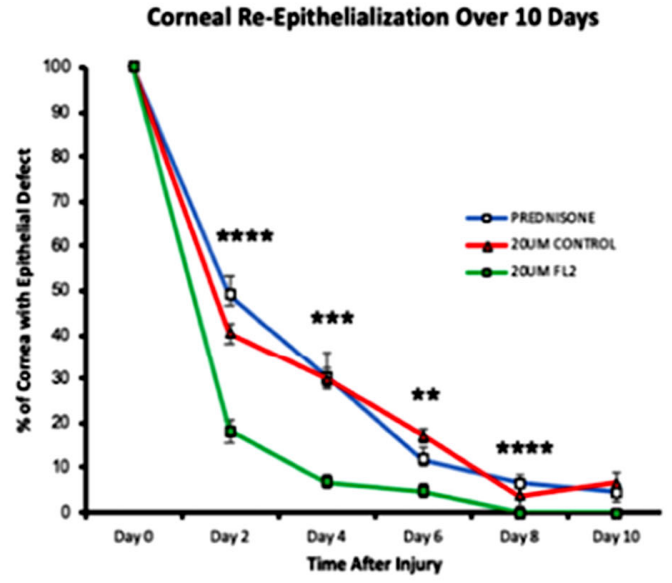

Figure 1. siRNA-mediated depletion of FL2 enhances corneal re-epithelialization. (A) Images of fluorescein-stained eyes day of and 8 days after injury. (B) Plots showing the kinetics of corneal re-epithelialization over 10 days with prednisone (blue line), $10 \mu \mathrm{M}$ control-NPsi (red line), and $10 \mu \mathrm{M}$ FL2-NPsi (green line). (C) Plots showing the kinetics of corneal re-epithelialization over 10 days with prednisone (blue line), $20 \mu \mathrm{M}$ control-NPsi (red line), and $20 \mu \mathrm{M}$ FL2-NPsi (green line). Data were pooled from multiple independent assays from corneas with alkaline injuries. The data for every time point were assessed using 1-way ANOVA and the levels of significance shown $(* * * *=p<0.0001$; ${ }^{* * *}=p<0.001 ;{ }^{* *}=p<0.01 ;{ }^{*}=p<0.05$; n.s., not significant).

was stained with neuronal specific rabbit polyclonal $\beta$-III tubulin antibody (BioLegend, San Diego, CA). Corneas were flat mounted on slides and imaged using an EVOS FL Auto Imaging System (ThermoFisher, Waltham, MA). Innervation of the cornea was defined as tortuous nerve endings organized in a clustered pattern originating from a single larger nerve and extending in three dimensions. Clusters were identified and counted manually from epifluorescence images of intact and hemisected corneal whole mounts. ${ }^{31}$

\section{siRNA Nanoparticle Synthesis}

Nanoparticles were prepared as follows ${ }^{12}: 500 \mu \mathrm{l}$ of Tetramethyl orthosilicate (TMOS) was hydrolyzed in the presence of $100 \mu \mathrm{l}$ of $1 \mathrm{mM} \mathrm{HCl}$ by sonication on ice for about 15 minutes, until a single phase formed. The hydrolyzed TMOS $(100 \mu \mathrm{l})$ was added to $900 \mu \mathrm{l}$ of $10 \mu \mathrm{M}$ of pooled siRNA against rat FL2 (siRNA from Sigma-Aldrich (St. Louis, MO): SASI_Rn02 00314854; SASI_Rn02 00314854; SASI_Rn02_00389576) or the negative control siRNA (Sigma, Universal Negative 
Table. Percentages of Corneal Surface Area With Epithelial Defect Over 14 Days After Alkaline Injury

Percentage of Cornea Surface Area with Epithelial Defect

\begin{tabular}{lcccccccc}
\cline { 2 - 8 } Treatment Group & Day 0 & Day 2 & Day 4 & Day 6 & Day 8 & Day 10 & Day 12 & Day 14 \\
\hline Prednisone & $100 \%$ & $49.2 \%$ & $30.4 \%$ & $11.9 \%$ & $6.5 \%$ & $4.4 \%$ & $0 \%$ & $0 \%$ \\
Empty NP & $100 \%$ & $39.1 \%$ & $21.8 \%$ & $17.0 \%$ & $7.5 \%$ & $4.6 \%$ & $0 \%$ & $0 \%$ \\
$1 \mu$ M control NP-si & $100 \%$ & $36.5 \%$ & $22.5 \%$ & $12.4 \%$ & $9.7 \%$ & $3.9 \%$ & $0 \%$ & $0 \%$ \\
$10 \mu$ M control NP-si & $100 \%$ & $48.0 \%$ & $23.7 \%$ & $15.7 \%$ & $8.2 \%$ & $5.1 \%$ & $0 \%$ & $0 \%$ \\
$20 \mu$ M control NP-si & $100 \%$ & $40.5 \%$ & $29.9 \%$ & $17.1 \%$ & $13.7 \%$ & $6.4 \%$ & $0 \%$ & $0 \%$ \\
$1 \mu$ M FL2 NP-si & $100 \%$ & $26.8 \%$ & $12.5 \%$ & $9.8 \%$ & $3.1 \%$ & $0 \%$ & $0 \%$ & $0 \%$ \\
$10 \mu$ M FL2 NP-si & $100 \%$ & $16.2 \%$ & $7.0 \%$ & $2.7 \%$ & $0.1 \%$ & $0 \%$ & $0 \%$ & $0 \%$ \\
$20 \mu$ M FL2 NP-si & $100 \%$ & $18.2 \%$ & $6.9 \%$ & $4.9 \%$ & $0 \%$ & $0 \%$ & $0 \%$ & $0 \%$ \\
\hline
\end{tabular}

A)

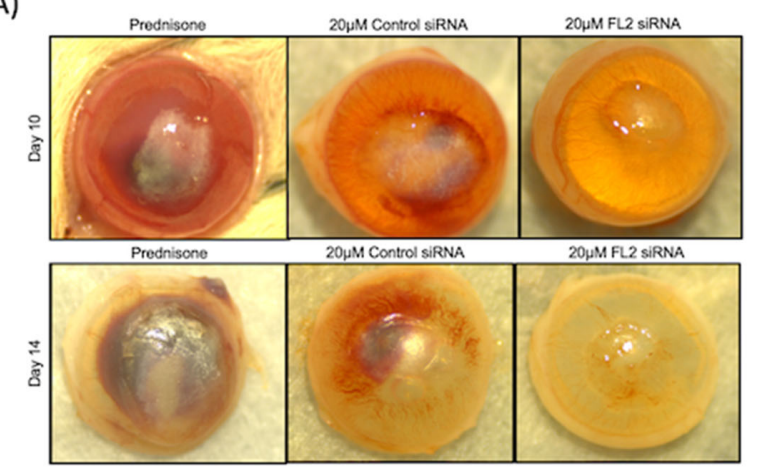

B)

\begin{tabular}{|rr|}
\hline Corneal Opacity Analysis & \\
Treatment Group & Opacity Score \\
PREDNISONE & 2.67 \\
EMPTY NP & 3.17 \\
\hline 1UM CONTROL NP-si & 1.83 \\
10UM CONTROL NP-si & 2.83 \\
\hline 20UM CONTROL NP-si & 2.17 \\
\hline 1UM FL2 NP-si & 1.67 \\
\hline 1OUM FL2 NP-si & 1.67 \\
\hline 2OUM FL2 NP-si & 1.17 \\
\hline
\end{tabular}

Figure 2. siRNA-mediated depletion of FL2 enhances corneal transparency in corneal tissue. (A) Corneal appearance at day 10 and day 14. In the prednisone-treated group, corneal tissue is cloudy with poor central corneal healing after alkali injury. Peripheral neovascularization is extensive. In the $20 \mu \mathrm{M}$ control-NPsi-treated group, corneal tissue is cloudy and exhibits extensive peripheral neovascularization. Some hemorrhage is seen behind the corneal tissue. In the $20 \mu \mathrm{M}$ FL2-NPsi-treated group, the corneal tissue is more transparent and less edematous with less peripheral neovascularization. Central corneal tissue healing appears improved compared to prednisone and controlNPsi-treated groups. (B) Table of corneal opacity scores after 14 days.

control B) solution containing $10 \mathrm{mM}$ phosphate, $\mathrm{pH}$ 7.4. A gel was formed within 10 minutes. The gel was frozen at $-80^{\circ} \mathrm{C}$ for 15 minutes and lyophilized. The dried sample was ground into a fine powder with a mortar and pestle. The nanoparticles were then resuspended in sterile PBS at an siRNA concentration of 1 , 10 , and $20 \mu \mathrm{M}$, and stored at $-80^{\circ} \mathrm{C}$ until immediately before use.

\section{Quantitative Polymerase Chain Reaction}

For in vivo knockdown experiments, RNA from four half-corneas per treatment group with an additional uninjured/untreated group (from the contralateral eye of the same rats) to serve as negative control were used. For in vitro knockdown experiments, rat B35 cells were treated for 24 hours with NPsi before harvesting and RNA extraction. The experiment was performed independently three times.
RNA was extracted with Trizol (Fisher, Hampton, $\mathrm{NH}$ ) by standard protocol. Two hundred to $300 \mathrm{ng}$ of RNA was reverse transcribed using the SSVilo IV kit (Invitrogen, Carlsbad, CA). PowerSYBR Green Master Mix (Invitrogen) was used for qPCR, using the 7300 Real-Time PCR system (Applied Biosystems); rat Fignl2: GAGTTGCTGCAGTGTGAATG and CTCTGTGCTTCTGTCTCTGT; rat b-actin: CGTTGACATCCGTAAAGACC (sense), and TCTCCTTCTGCATCCTGTCA (antisense). Results were quantified using the comparative $2^{-\mathrm{DDC} t}$ method.

\section{Statistical Analysis}

All data analysis in this study was carried out using GraphPad Prism 6 or Microsoft Excel (version 16.16.18). The samples and animals were allocated to experimental groups and processed randomly. All 
A)

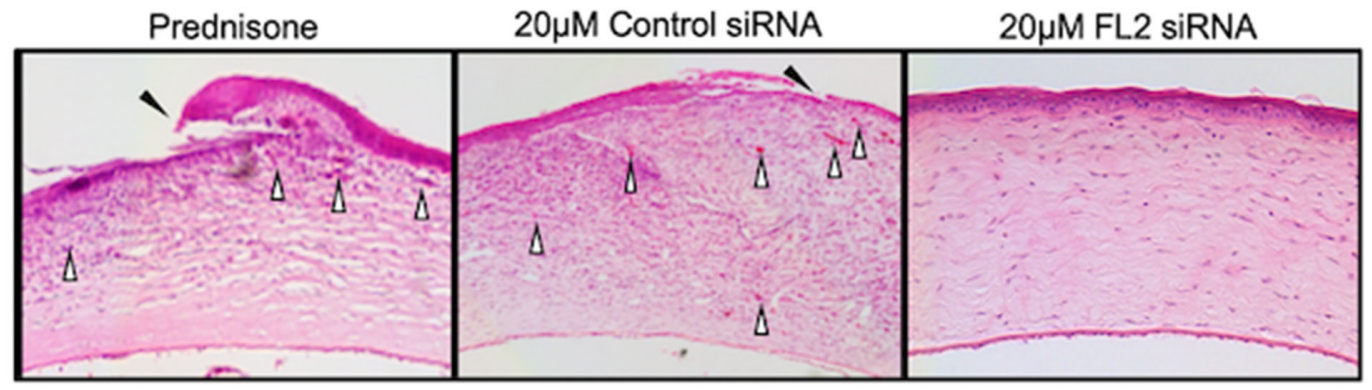

B)

\begin{tabular}{|c|c|c|c|c|}
\hline \multicolumn{5}{|c|}{ Histopathology Analysis } \\
\hline Treatment Group & Stromal Edema & Stromal Inflammation & Stromal Neovascularization & $\begin{array}{l}\text { AC Inflammation/ } \\
\text { Retrocorneal membrane }\end{array}$ \\
\hline PREDNISONE & 2.92 & 2.67 & 3.33 & 2.33 \\
\hline EMPTY NP & 2.75 & 2.91 & 2.91 & 0.83 \\
\hline IUM CONTROL NP-Si & 2.67 & 2.42 & 2.58 & 1.92 \\
\hline IOUM CONTROL NP-SI & 2.67 & 2.58 & 2.83 & 0.17 \\
\hline 2OUM CONTROL NP-si & 2.58 & 2.67 & 2.67 & 1.8 \\
\hline IUM FL2 NP-si & 2.5 & 2.4 & 2.5 & 1.1 \\
\hline $10 U M$ FL2 NP-si & 2.25 & 2 & 2.08 & 0 \\
\hline 20 UM FL2 NP-si & 2 & 1.83 & 1.83 & 0 \\
\hline
\end{tabular}

C)

\begin{tabular}{|r|r|r|r|}
\hline & Immunohistochemical Analysis & \\
\hline Treatment Group & $\mathrm{CO} 31$ & Neutrophils & Macrophages \\
\hline PREDNISONE & 0 & 2 & 1 \\
EMPTY NP & 0.5 & 3 & 1 \\
\hline 1UM CONTROL NP-si & 1 & 3 & 2 \\
10UM CONTROL NP-si & 0.5 & 2.5 & 1.5 \\
\hline 2OUM CONTROL NP-si & 0 & 3 & 1 \\
\hline 1UM FL2 NP-si & 0 & 3 & 1 \\
\hline 1OUM FL2 NP-si & 1 & 2 & 1.5 \\
\hline 20UM FL2 NP-si & 1 & 2 & 1 \\
\hline
\end{tabular}

Figure 3. FL2-NPsi treatment stimulates healing in the rat alkali burn injury model. (A) Following 14 days of treatment, sections of corneal tissues were stained with H\&E and examined. Representative images shown for rats treated with Prednisone, $20 \mu M$ control-NPsi, or $20 \mu \mathrm{M}$ FL2-NPsi. The corneal epithelium layer is poorly healed, thinned, and irregular with superficial keratinization (black arrowheads) in prednisone and control-NPsi-treated groups. The corneal stroma is extremely edematous, and collagen lamella is disorganized and infiltrated with many inflammatory cells and extensive neovascularization (white arrowheads). In the FL2-NPsi-treated group, the corneal edema is less pronounced and the epithelium is thicker and more regular compared to prednisone or control-NPsi-treated groups. The corneal stroma shows less inflammatory cell infiltration and less neovascularization. Data is representative of $\geq 3$ independent experiments. (B) Table of histopathologic scores after 14 days. (C) Table of immunohistochemical scores.

in vitro experiments represented multiple independent experiments conducted in triplicate. The in vivo experiments were performed with 2 to 6 rats for each condition. All data are represented as means \pm SEM. Statistical analyses for the above characteristics were performed using the unpaired Student's $t$-test (for comparing two distributions) and 1-way ANOVA for more than two distributions. Differences were considered statistically significant at a $P$ value of $<0.05$.

\section{Results}

Alkaline chemical injuries were induced onto the right cornea of rats, which were subsequently treated with prednisolone, empty nanoparticles, control-NPsi $(1 \mu \mathrm{M}, 10 \mu \mathrm{M}$, and $20 \mu \mathrm{M})$ and FL2-NPsi $(1 \mu \mathrm{M}$, $10 \mu \mathrm{M}$, and $20 \mu \mathrm{M})$. FL2-NPsi-treated groups demonstrated significantly greater corneal re-epithelialization rates when compared to the prednisone and their 
A)

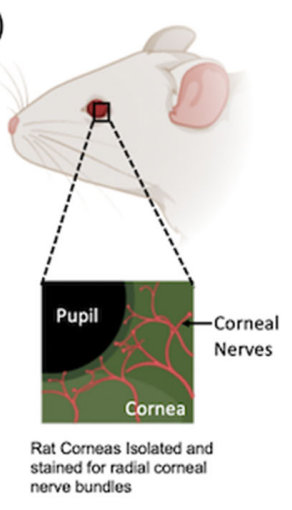

B)

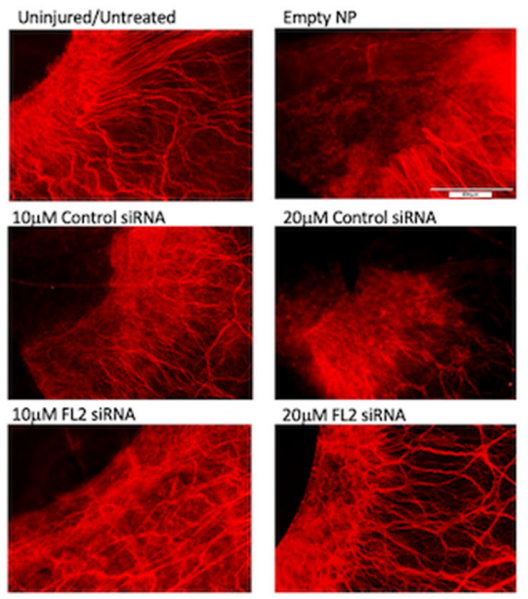

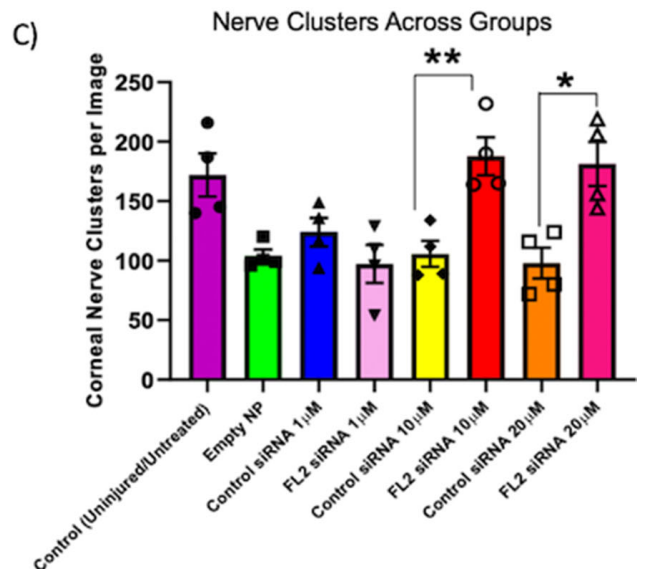

Figure 4. Corneal nerve assessment using $\boldsymbol{\beta}$-III tubulin antibody. (A) Schematic of experiment for corneal nerve assessment. Corneal samples were derived from rats treated/untreated with siRNA NPs. Whole mount corneas were imaged after staining for corneal nerve bundles. (B) Corneas stained with $\beta$-III tubulin antibody in corneal tissue reveal that empty NP and control-NPsi-treated eyes demonstrate similar nerve densities, while uninjured/untreated and FL2-NPsi-treated eyes exhibit comparable nerve densities. Red $=\beta$-III Tubulin. (C) Quantification of nerve clusters across treatment groups reveal that the $10 \mu \mathrm{M}$ and $20 \mu \mathrm{M}$ FL2-NPsi-treated corneas demonstrate a greater number of nerve clusters as compared with the control groups, and is comparable to that of uninjured/untreated corneas.

respective control concentration groups (Fig. 1, Table). Specifically, 10 and $20 \mu \mathrm{M}$ FL2-NPsi were determined to be the most efficacious concentrations in reducing time to corneal re-epithelialization throughout the healing process $(p=0.0003$ at day $4 ; p<0.0001$ at day 8 ).

The degree of corneal opacity was scored from a scale of 0 to 4 (Supplement Fig. S1). The prednisone, empty NP, $1 \mu \mathrm{M}$ control-NPsi, $10 \mu \mathrm{M}$ control-NPsi, $20 \mu \mathrm{M}$ control-NPsi groups scored between 3 and 4 across all eyes. The $1 \mu \mathrm{M}, 10 \mu \mathrm{M}$ FL2-NPsi, and $20 \mu \mathrm{M}$ FL2-NPsi groups, however, scored between 1 and 2. When comparing control and FL2 treatment groups by concentration, the $20 \mu \mathrm{M}$ FL2-NPsi-treated eyes demonstrated statistically significantly decreased opacity with less peripheral neovascularization and central edema when compared to those treated with $20 \mu \mathrm{M}$ of control-NPsi (Fig. 2, Supplement Table S2). However, the $10 \mu \mathrm{M}$ and $1 \mu \mathrm{M}$ FL2-NPsi groups were not significantly different from their control-NPsi counterparts.

After 14 days, all layers of corneal tissue were compared in a blinded fashion, including for epithelial healing (structure and organization), arrangement of stromal collagen lamellae, and presence of edema, inflammatory cells, and vascularization. Histopathologic analysis by a blinded ocular pathologist using a scoring system of 0 to $4+$, with 0 being absent and $4+$ being most severe, revealed 2 to $3+$ stromal edema, inflammation, and neovascularization and $2+$ retrocorneal membrane in control groups. In contrast, the FL2-NPsi-treated population exhibited fewer inflammatory cells and less neovascularization, with stromal edema, inflammation, and neovascularization scored as 1 to $2+$ in the absence of retrocorneal membranes. In fact, statistical analysis reveals that the $10 \mu \mathrm{M}$ and $20 \mu \mathrm{M}$ FL2-NPsi-treated groups had a statistically significant milder degree of stromal neovascularization and retrocorneal membrane. The degree of stromal edema and stromal inflammation was not found to be statistically significantly different. Immunohistochemical analysis did not yield any significant differences between groups, possibly due to a variety of technical challenges including elevated background signal (likely due to the injury). Further studies with more markers will need to be performed in future experiments (Fig. 3, Supplement Table S3).

As there is less tissue in the wounded zone, confirming knockdown in vivo proved to be technically difficult. There is no reliable antibody against the rat homolog of FL2, so Western blotting to confirm knockdown was not an option. For these reasons and despite multiple attempts, confirming knockdown in vivo was not achieved. FL2-NPsi induced knockdown was, however, confirmed in vitro using B35 rat neuroblastoma cells (Supplement Fig. S4).

Last, preliminary toxicology studies using quantitative microscopy suggest that alkaline chemical injuries are deleterious to corneal nerves, but FL2-NPsi is not. In fact, the $10 \mu \mathrm{M}$ and $20 \mu \mathrm{M}$ FL2-NPsi-treated groups had a greater number of nerve clusters as compared with control and untreated groups, demonstrating a similar profile to the uninjured/untreated 
A)
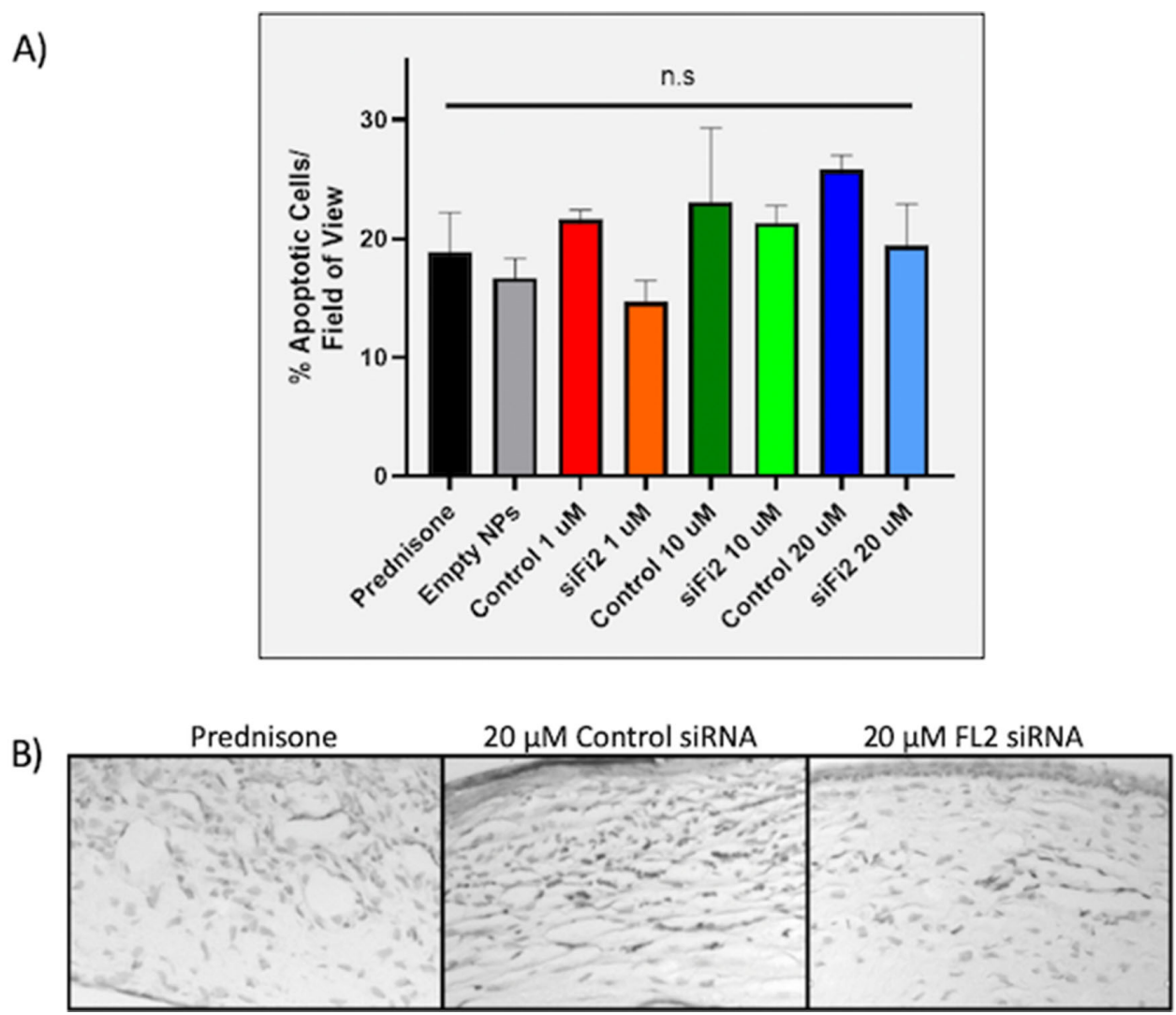

Figure 5. Apoptosis in corneal tissue using TUNEL assay. (A) Prednisone, control-NPsi, and FL2-NPsi-treated corneas exhibit no statistically significant difference in the number of apoptotic cells after alkaline chemical injury. n.s., not significant. (B) Apoptotic cells in prednisone, $20 \mu \mathrm{M}$ control-NPsi, and $20 \mu \mathrm{M}$ FL2-NPsi-treated corneas.

negative control cornea (Fig. 4, Supplement Fig. S5), whereas empty nanoparticle and control-NPsi-treated groups demonstrated significantly reduced nerve densities. In addition, there was not a statistically significant difference in the number of apoptotic cells among prednisone, control, or FL2-NPsi-treated groups on the TUNEL assay ( $p=0.917$; Fig. 5, Supplement Table S6), suggesting that FL2-NPsi is not toxic to corneal tissues.

\section{Discussion}

In recent years, there have been multiple investigations into harnessing the intracellular cytoskeleton, particularly microtubules, in tissue repair. Along with their structural role, microtubules are an important substrate for many of the molecular motor proteins responsible for intracellular transport. Regulation of the dynamic properties of microtubules are critically important (especially near the cell periphery) for the capacity of cells to close wounds. Our previous work screening biological agents for cell migration pheno- types identified a novel regulator of the microtubule cytoskeleton, termed FL2, as an important negative regulator of cell migration. ${ }^{12}$ The siRNA-mediated depletion of FL2 results in a significant increase in the velocity and directionality of cells in vitro. ${ }^{12}$ Moreover, we found that in vivo depletion of FL2 strongly promoted wound closure and repair. ${ }^{12,13}$ FL2 depletion enhanced healing by inducing a targeted, transient disruption of cortical microtubules within the leading edge of cells. ${ }^{12}$ Charafeddine et al. (2015) translated these basic cell biology findings into an in vivo murine model of wound healing using a nanoparticle formulation to deliver siRNA to wounded skin. Here, we applied the same core nanoparticle technology to enhance corneal repair.

Exposure of the cornea to alkaline solutions, particularly ammonia, can result in near immediate damage to the corneal basement membrane, stromal keratocytes and nerve endings, endothelium, lens epithelium, and vascular endothelium of the conjunctiva, episclera, iris, and ciliary body. The penetration of ammonia can cause saponification of cell membranes leading to cell death, hydration of glycosaminoglycans leading to reduced stromal clarity, hydration of collagen fibrils 
leading to alterations of the trabecular meshwork, and the release of prostaglandins..$^{32-34}$ As a result, significant inflammation typically occurs and can dramatically slow the rate of epithelial cell migration into the wound zone. ${ }^{35-37}$

Recovery toward an intact corneal epithelium is noted to be the most important determinant for a favorable outcome following chemical injury, ${ }^{4}$ because its presence signals a positive feedback loop that limits the production of collagenase in the stroma after reepithelialization, thereby maintaining corneal transparency. ${ }^{38-40}$ Studies have demonstrated that an intact epithelium may also be critical in preventing additional rounds of inflammation. When an epithelial defect remains after 7 days, a second wave of inflammation begins and persists. ${ }^{41,42}$ Therefore, identifying a factor that can expedite the migration of cells to reform an intact corneal epithelium can dramatically reduce inflammatory complications and improve outcomes.

When considering corneal re-epithelization, the $10 \mu \mathrm{M}$ and $20 \mu \mathrm{M}$ FL2-NPsi groups demonstrated the greatest rates of epithelial healing. In contrast, the 1 $\mu \mathrm{M}$ FL2-NPsi-treated group performed worse than the 10 and $20 \mu \mathrm{M}$ concentrations, most likely because 1 $\mu \mathrm{M}$ is too low a concentration to produce a significant effect on gene expression. Still, all FL2 NPsi-treated groups showed a greater rate of re-epithelialization compared to control-NPsi, empty NP, and prednisonetreated groups (see Fig. 1, Table).

In addition to efficacy, the safety of FL2-NPsi is an important consideration for clinical translation. Interestingly, our preliminary toxicology evaluation using quantitative microscopy to assess for nerve damage found that FL2-treated corneas exhibited similar profiles to uninjured/untreated negative controls. This finding suggests that FL2 may not only be nontoxic to corneal nerves, but may also play a role in nerve restoration after alkaline chemical injury. In fact, a recent study by Baker et al., found that FL2 knockdown had a neuro-restorative effect on injured cavernous nerves in a rodent model. ${ }^{43}$ The increase in the number of nerve clusters observed in the $10 \mu \mathrm{M}$ and $20 \mu \mathrm{M}$ of FL2-NPsi groups warrants further studies to assess for the possible neuro-restorative potential of this treatment (see Fig. 4, see Supplement Fig. S5).

The current use of siRNA to treat ocular maladies presents an area ripe for future development. Because the eye is a confined compartment and easily accessible for topical treatment, siRNA may be particularly promising. ${ }^{44}$ Recent approaches using siR NAs to downregulate the expression of genes associated with proliferation, fibrosis, or inflammation (e.g. CAP37, Caveolin-1, TGFB1, TGFBR2, CTGF, and VEGFR1) have shown a range of promising preclinical data. However, these targeted proteins are involved in known cancer pathways, limiting clinical translation and underscoring the need to identify a new gene target. ${ }^{45-48}$

In summary, rapid recruitment of epithelial cells responsible for closing wounds and stabilizing the corneal surface may be key in wound healing. By enhancing cell motility, wound healing can occur more rapidly and with high fidelity to the original tissue. This can have several profound effects on recovery, including reduced scarring, pain, risk of infection, and improved vision and restoration of corneal architecture.

\section{Acknowledgments}

The authors thank Peng Guo, PhD, for his help with analytical imaging.

Supported by grant NIH/NEI Phase 1 STTR \#1R43AR1234456-01A1.

Disclosure: J. Wang, MicroCures, Inc. (E); A. Dey, MicroCures, Inc. (E); A.H. Kramer, MicroCures, Inc. (E); Y. Miao, None; J. Liu, None; L. Baker, MicroCures, Inc. (E); J.M. Friedman, MicroCures, Inc. (I); P. Nacharaju, None; R.S. Chuck, MicroCures, Inc. (C); C. Zhang, None; D.J. Sharp, MicroCures, Inc. $(\mathrm{F}, \mathrm{I}, \mathrm{C}, \mathrm{P})$

${ }^{*} \mathrm{CZ}$ and DJS contributed equally to this paper and should be considered co-senior authors.

\section{References}

1. Solano JJ. Ocular Burns and Chemical Injuries. 2019 January 31, 2019. Available from: https:// emedicine.medscape.com/article/798696-clinical.

2. Singh P, Tyagi M, Kumar Y, Gupta KK, Sharma PD. Ocular chemical injuries and their management. Oman J Ophthalmol. 2013;6(2):83-86.

3. Phillips K, Arffa R, Cintron C, et al. Effects of prednisolone and medroxyprogesterone on corneal wound healing, ulceration, and neovascularization. Arch Ophthalmol. 1983;101(4):640-643.

4. Wagoner MD. Chemical injuries of the eye: current concepts in pathophysiology and therapy. Surv Ophthalmol. 1997;41(4):275-313.

5. Hanna C, Bicknell DS, O'Brien JE, Cell turnover in the adult human eye. Arch Ophthalmol. 1961;65:695-698. 
6. Copeland RA, Afshari N, Dohlman CH. Copeland and Afshari's Principals and Practice of Cornea. City of Knowledge, Clayton, Republic of Panama: Jaypee-Highlights Medical Publishers, Inc.; 2013;704.

7. Anderson C, Zhou Q, Wang S. An alkali-burn injury model of corneal neovascularization in the mouse. J Vis Exp. 2014;86:51159.

8. Bai JQ, Qin HF, Zhao SH. Research on mouse model of grade II corneal alkali burn. Int J Ophthalmol. 2016;9(4):487-490.

9. Xiao O, Xie X, Lin B, Yin X, Pi R, Zhou S. Minocycline inhibits alkali burn-induced corneal neovascularization in mice. PLoS One. 2012;7(7):e41858.

10. Ashby BD, Garrett Q, Willcox M. Corneal Injuries and wound healing - review of processes and therapies. Austin J Clin Ophthalmol. 2014;1(4):1017.

11. Turner CT, McInnes SJP, Melville E, Cowin AJ, Voelcker NH. Delivery of flightless I siRNA from porous silicon nanoparticles improves wound healing in mice. Adv Healthc Mater. 2016;2(12):23392346.

12. Charafeddine RA, Makdisi J, Schairer D, et al. Fidgetin-like 2: a microtubule-based regulator of wound healing. $J$ Invest Dermatol. 2015;135(9):2309-2318.

13. O'Rourke BP, Kramer AH, Cao LL, et al. Fidgetin-like 2 siRNA enhances the wound healing capability of a surfactant polymer dressing. $A d v$ Wound Care (New Rochelle). 2018;8(3):91-100.

14. Martinez T, González MV, Roehl I, Wright N, Pañeda C, Jiménez AI. In vitro and in vivo efficacy of SYL040012, a novel siRNA compound for treatment of glaucoma. Mol Ther. 2014;22(1):8191.

15. Behlke MA. Chemical modification of siRNAs for in vivo use. Oligonucleotides. 2008;18(4):305-319.

16. Li Z, Duan F, Lin L, Huang Q, Wu K. A new approach of delivering siRNA to the cornea and its application for inhibiting herpes simplex keratitis. Curr Mol Med. 2014;14(9):1215-1225.

17. Schiroli D, Gómara MJ, Maurizi E, et al., Topical delivery of siRNA and gene silencing in intact corneal epithelium using a modified cellpenetrating peptide. Mol Ther Nucleic Acids. 2019;17:891-906.

18. Gordon SR, Staley CA, Role of the cytoskeleton during injury-induced cell migration in corneal endothelium. Cell Motil Cytoskeleton. 1990;16(1):47-57.

19. Gordon S, Buxar R, Inhibition of cytoskeletal reorganization stimulates actin and tubulin syntheses during injury-induced cell migration in the corneal endothelium. J Cell Biochem. 1998;67:409421.

20. Wang J, Lin A, Lu L. Effect of EGF-induced HDAC6 activation on corneal epithelial wound healing. Invest Ophthalmol Vis Sci. 2010;51(6):2943-2948.

21. Pothula S, Bazan HE, Chandrasekher G. Regulation of $\mathrm{Cdc} 42$ expression and signaling is critical for promoting corneal epithelial wound healing. Invest Ophthalmol Vis Sci. 2013;54(8):53435352.

22. Minns MS, Teicher G, Rich CB, Trinkaus-Randall V. Purinoreceptor P2X7 regulation of $\mathrm{Ca}(2+)$ mobilization and cytoskeletal rearrangement is required for corneal reepithelialization after injury. Am J Pathol. 2016;186(2):285-296.

23. Alster Y, Varssano D, Loewenstein A, Lazar M. Delay of corneal wound healing in patients treated with colchicine. Ophthalmology. 1997;104(1):118 119.

24. Leibovitch I, Alster Y, Lazar M, Langevitz P, Livneh A, Loewenstein A. Corneal wound healing in a patient treated with colchicine for familial Mediterranean Fever (FMF). Rheumatology (Oxford). 2003;42(8):1021-1022.

25. Roll-Mecak A, McNally FJ. Microtubule-severing enzymes. Curr Opin Cell Biol. 2010;22(1):96-103.

26. Roll-Mecak A, Vale RD. Making more microtubules by severing: a common theme of noncentrosomal microtubule arrays? $J$ Cell Biol. 2006;175(6):849-851.

27. Sharp DJ, Ross JL. Microtubule-severing enzymes at the cutting edge. $J$ Cell Sci. 2012;125(Pt 11):2561-2569.

28. Bartlett DW, Davis ME. Insights into the kinetics of siRNA-mediated gene silencing from live-cell and live-animal bioluminescent imaging. Nucleic Acids Res. 2006;34(1):322-333.

29. Allergan, Pred Forte 1\% w/v, Eye Drops Suspension. 2003. Available at: https://www.medicines. org.uk/emc/product/1354/smpc.

30. Allergan, Product Monograph, Ocuflox. 1994. Available at: https://allergan-web-cdn-prod.azure edge.net/allergancanadaspecialty/allergancanadas pecialty/media/actavis-canada-specialty/en/ products/pms/2018-02-06-ocuflox-pm_en.pdf.

31. Fink DM, Connor AL, Kelley PM, Steele MM, Hollingsworth MA, Tempero RM. Nerve growth factor regulates neurolymphatic remodeling during corneal inflammation and resolution. PLoS One. 2014;9(11):e112737.

32. Grant WM, Kern HL. Action of alkalies on the corneal stroma. AMA Arch Ophthalmol. 1955;54(6):931-939. 
33. Eslani M, Baradaran-Rafii A, Movahedan A, Djalilian AR. The ocular surface chemical burns. J Ophthalmol. 2014;2014:196827.

34. Schrage NF, Langefeld S, Zschocke J, Kuckelkorn R, Redbrake C, Reim M. Eye burns: an emergency and continuing problem. Burns. 2000;26(8):689699.

35. Wagoner MD, Kenyon KR, Gipson IK, Hanninen LA, Seng WL. Polymorphonuclear neutrophils delay corneal epithelial wound healing in vitro. Invest Ophthalmol Vis Sci. 1984;25(10):1217-1220.

36. Watanabe M, Kondo S, Mizuno K, et al. Promotion of corneal epithelial wound healing in vitro and in vivo by annexin A5. Invest Ophthalmol Vis Sci. 2006;47(5):1862-1868.

37. Oh SY, Choi J-S, Kim E-J, Chuck RS, Park CY. The role of macrophage migration inhibitory factor in ocular surface disease pathogenesis after chemical burn in the murine eye. Mol Vis. 2010;16:2402-2411.

38. Perry HD, Hodes LW, Seedor JA, Donnenfeld ED, McNamara TF, Golub LM. Effect of doxycycline hyclate on corneal epithelial wound healing in the rabbit alkali-burn model. Preliminary observations. Cornea. 1993;12(5):379-382.

39. Sudbeck BD, Pilcher BK, Welgus HG, Parks WC. Induction and repression of collagenase-1 by keratinocytes is controlled by distinct components of different extracellular matrix compartments. J Biol Chem. 1997;272(35):22103-22110.

40. Inoue M, Kratz G, Haegerstrand A, StåhleBäckedahl M. Collagenase expression is rapidly induced in wound-edge keratinocytes after acute injury in human skin, persists during healing, and stops at re-epithelialization. $J$ Invest Dermatol. 1995;104(4):479-483.

41. Paterson CA, Williams RN, Parker AV. Characteristics of polymorphonuclear leukocyte infiltration into the alkali burned eye and the influence of sodium citrate. Exp Eye Res. 1984;39(6):701708.

42. Katzman LR, Jeng BH. Management strategies for persistent epithelial defects of the cornea. Saudi $J$ Ophthalmol. 2014;28(3):168-172.

43. Baker L, Tar M, Villegas G, et al. Fidgetinlike 2 is a novel negative regulator of axonal growth and can be targeted to promote functional nerve regeneration after injury. bioRxiv, 2020;2020.03.19.999508.

44. Guzman-Aranguez A, Loma P, Pintor J. Smallinterfering RNAs (siRNAs) as a promising tool for ocular therapy. Br J Pharmacol. 2013;170(4):730 747.

45. Griffith GL, Kasus-Jacobi A, Lerner MR, Pereira HA. Corneal wound healing, a newly identified function of CAP37, is mediated by protein kinase C delta (PKC $\delta$ ). Invest Ophthalmol Vis Sci. 2014;55(8):4886-4895.

46. Rhim JH, Kim JH, Yeo E-J, Kim JC, Park SC. Caveolin-1 as a novel indicator of wound-healing capacity in aged human corneal epithelium. Mol Med. 2010;16(11-12):527-534.

47. Yousuf MA, Zhou X, Mukherjee S, et al. Caveolin1 associated adenovirus entry into human corneal cells. PLoS One. 2013;8(10):e77462.

48. Saika S. TGF-beta signal transduction in corneal wound healing as a therapeutic target. Cornea. 2004;23(8 Suppl):S25-S30. 